\title{
molecular microbiology
}

\section{A 20 residue peptide of the inner membrane protein OutC mediates interaction with two distinct sites of the outer membrane secretin OutD and is essential for the functional type II secretion system in Erwinia chrysanthemi.}

\begin{tabular}{|c|c|}
\hline Journal: & Molecular Microbiology \\
\hline Manuscript ID: & MMI-2009-09558.R2 \\
\hline Manuscript Type: & Research Article \\
\hline $\begin{array}{r}\text { Date Submitted by the } \\
\text { Author: }\end{array}$ & 17-Mar-2010 \\
\hline Complete List of Authors: & $\begin{array}{l}\text { Login, Frédéric; Université Lyon1, MAP, UMR } 5240 \text { CNRS-Université } \\
\text { Lyon1, INSA Lyon, BCS } \\
\text { Fries, Markus; Queen Mary University of London, School of } \\
\text { Biological and Chemical Sciences } \\
\text { Wang, Xiaohui; INSA de Lyon, MAP, UMR } 5240 \text { CNRS-Université } \\
\text { Lyon1-Insa Lyon-BCS } \\
\text { Pickersgill, Richard; Queen Mary University of London, School of } \\
\text { Biological and Chemical Sciences } \\
\text { Shevchik, Vladimir; vladimir.shevchik@insa-lyon.fr, MAP, UMR } 5240\end{array}$ \\
\hline Key Words: & type II secretion system, secretin, GspC, GspC-GspD interaction \\
\hline
\end{tabular}

\section{ScholarONE"


1 A 20 residue peptide of the inner membrane protein OutC mediates interaction with two

2 distinct sites of the outer membrane secretin OutD and is essential for the functional

3 type II secretion system in Erwinia chrysanthemi.

4

5 Frédéric H. Login ${ }^{1}$, Markus Fries ${ }^{2}$, Xiaohui Wang ${ }^{1}$, Richard W. Pickersgill ${ }^{2}$ and Vladimir E.

$6 \quad$ Shevchik $^{1 *}$

7 1: Université de Lyon, F-69003, Université Lyon 1, Lyon, F-69622, INSA-Lyon,

8 Villeurbanne, F-69621, CNRS, UMR5240, Microbiologie Adaptation et Pathogénie, Lyon, F-

9 69622, France

10 2: School of Biological and Chemical Sciences, Queen Mary University of London, London

11 E1 4NS, UK

12

$13 *$ Corresponding author: vladimir.shevchik@insa-lyon.fr

14

Tel: (33) 472445827

15

Fax: (33) 472431584

16

17 Running title: Peptide of OutC interacts with secretin

18

19 Key words: type II secretion system, secretin, GspC, GspC-GspD interaction 


\section{Summary}

2 The type II secretion system (T2SS) is widely exploited by proteobacteria to secrete enzymes

3 and toxins involved in bacterial survival and pathogenesis. The outer membrane pore formed

4 by the secretin OutD and the inner membrane protein OutC are two key components of the

5 secretion complex, involved in secretion specificity. Here, we show that the periplasmic

6 regions of OutC and OutD interact directly and map the interaction site of OutC to a 20

7 residue peptide named OutCsip (secretin interacting peptide, residues 139 to 158). This

8 peptide interacts in vitro with two distinct sites of the periplasmic region of OutD, one located

9 on the N0 subdomain and another overlapping the N2-N3' subdomains. The two interaction

10 sites of OutD have different modes of binding to OutCsip. A single substitution, V143S,

11 located within OutCsip prevents its interaction with one of the two binding sites of OutD and

12 fully inactivates the T2SS. We show that the N0 subdomain of OutD interacts also with a

13 second binding site within OutC located in the region proximal to the TMS. We suggest that

14 successive interactions between these distinct regions of OutC and OutD may have functional

15 importance in switching the secretion machine. 


\section{Introduction}

2 The type II secretion system (T2SS) is widely exploited by proteobacteria to secrete various 3 proteins involved in pathogenesis and environmental survival (Filloux 2004; Cianciotto, 2005; Evans et al., 2008). Secretion by the T2SS is a two-step process. The proteins to be secreted (exoproteins) are synthesized with an $\mathrm{N}$-terminal signal sequence and they cross the cytoplasmic membrane, either by the Sec or by the Tat translocon (Pugsley et al., 1991; Voulhoux et al., 2001). Once in the periplasm, the exoproteins are folded and then translocated across the outer membrane by the T2SS machinery that is composed of 12 to 15 proteins, also referred to as Gsp (general secretory pathway). The individual T2SS components are integrated in, or associated with, either the cytoplasmic or the outer membrane. The phytopathogenic bacterium Erwinia chrysanthemi possesses two independent T2SS, Out and Stt (Bouley et al., 2001; Ferrandez and Condemine, 2008). At least ten pectinases and a cellulase are secreted by the Out system (Kazemi-Pour et al., 2004). Two proteins, OutD and OutC, have been shown to be involved in the specificity of the secretion system, probably by direct recognition of exoproteins (Lindeberg et al., 1996; Shevchik et al., 1997; Bouley et al., 2001).

The secretin OutD is the integral outer membrane component of the T2SS. Secretins are involved in several systems of macromolecular transport through the outer membrane (Genin and Boucher, 1994). This channel-forming protein forms toroidal structures composed of 12 to 14 subunits (Linderoth et al., 1997; Bitter et al., 1998; Nouwen et al., 2000; Burghout et al., 2004). The periplasmic N-terminal domain of the secretins is more variable and confers specificity to the secretion (Daefler et al., 1997; Bouley et al., 2001). Direct interaction of the secretin OutD with the secreted exoproteins has also been demonstrated (Shevchik et al., 1997). The N-terminal domain of the secretin from enterotoxigenic E. coli (strain H10407)

24 (45\% amino acid identity with OutD) consists of four subdomains (N0 to N3) (Korotkov et al., 2009). The N0 subdomain has the same fold as the signalling domain of TonB-dependent 
1 receptors. The subdomains $\mathrm{N} 1$ and $\mathrm{N} 2$ exhibit a fold similar to the $\mathrm{KH}$ domains that is also

2 present in ring-forming proteins of the type III secretion system. The C-terminal domain of the

3 secretins is more conserved and is involved in multimerization and channel formation (Brok

4 et al., 1999; Nouwen et al., 2000; Chami et al., 2005). In certain T2SS, some accessory

5 components, the pilotin OutS and OutB, improve the maturation and targeting of the secretin

6 (Hardie et al., 1996; Shevchik and Condemine, 1998; Condemine and Shevchik, 2000). In the

7 absence of the pilotin, the secretin mislocalizes to the inner membrane (Guilvout et al., 2006).

OutC is the bitopic inner membrane protein possessing a large periplasmic region with

9 a C-terminally located PDZ domain (Bleves et al., 1996; Thomas et al., 1997; Korotkov et al., 2006). The PDZ domain of OutC appears to be involved in substrate recognition, as shown by swapping the PDZ domains of OutC from E. chrysanthemi and E. carotovora that switches the specificity of the secretion system (Bouley et al., 2001). The transmembrane segment (TMS) of OutC drives the protein multimerization (Login and Shevchik, 2006). GspC appears to be a less conserved component of the T2SS, only a part of the periplasmic region, the socalled homology region (HR) being highly conserved (Gérard-Vincent et al., 2002).

How the individual components of the T2SS interact to form a highly organized multiprotein machine is not well understood. It has been suggested that GspC and GspD could interact to provide the structural and functional integrity of the secretion machinery across the two membranes of the Gram-negative bacterium (Lindeberg et al., 1996; Shevchik et al., 1997). Such an interaction could also be involved in substrate recognition, the triggering of 21 pilus formation, and pore opening. Previous studies in Klebsiella oxytoca and Pseudomonas aeruginosa indirectly implicated a GspC-GspD interaction, but failed to detect it directly (Possot et al., 1999; Bleves et al., 1999). An interaction between XpsD and XpsN (GspD and GspC of Xanthomonas campestris) has been observed by cross-linking and pull-down assays 
1 the formation of this complex. However, the study of Vibrio vulnificus T2SS demonstrated a 2 strong interaction in vitro between the $\mathrm{N}$-domain of GspD and the HR-domain of the 3 periplasmic region of GspC (Korotkov et al., 2006). Conversely, the yeast two-hybrid assay 4 did not reveal any interaction between these regions of OutC and OutD (Douet et al., 2004). 5 Thus, the experimental data on the GspC-GspD interaction remains puzzling. One reason for 6 this may be the different experimental approaches used. In vivo, GspC and GspD probably 7 also interact with other components of the T2SS and with exoproteins. Some data suggest that 8 the periplasmic region of GspC proximal to the TMS interacts with the GspL-GspM 9 subcomplex (Lee et al., 2004; Gérard-Vincent et al., 2002; Possot et al., 2000). An interaction between the secretin GspD and the pseudopilins GspG and GspJ has also been detected by 11 cross-linking and by yeast two hybrid assays (Douet et al., 2004; Lee et al., 2005). In this study, we examined the interaction between the secretin OutD and an inner membrane component of the T2SS, OutC, using both in vitro and in vivo approaches. We show that a short segment of the periplasmic region of OutC, which is located within the Cterminal part of the HR domain, interacts with two distinct regions of the N-domain of OutD. Mutagenesis analysis shows that this segment of OutC is critical for the function of the T2SS. We also show that OutD interacts with a second binding site within OutC located in the region proximal to the TMS

\section{Results}

\section{Co-purification of OutD and GST-OutC from a functional T2SS}

22 Proteins interacting with OutC in vivo were identified by co-purification exploiting GSTtagged OutC as affinity bait. When the pTdB-GoC plasmid expressing gst-outC was introduced into the outC deficient strain E. chrysanthemi A3618, pectate lyase secretion was 
1 carrying pTdB-GoC was equal to that of chromosomally encoded OutC in the parental strain

2 A1077 as estimated using immunoblotting with OutC-antibodies (not shown). We conclude that GST-OutC is correctly integrated into the T2SS to form a functional secretion complex.

E. chrysanthemi A3618 $\Delta$ outC cells expressing either GST-OutC or GST alone were grown to a mid exponential phase and then used for affinity chromatography on Glutathione

Sepharose. Bound proteins were eluted from the resin and analyzed by 2D-gel electrophoresis

(Fig. S1). As expected a large quantity of GST (Fig. S1A) or of GST-OutC and a $31 \mathrm{kDa}$ species reactive with anti-GST (Fig. S1B) were detected. Importantly, a series of intense spots of about $70 \mathrm{kDa}$ were also observed in A3618 expressing GST-OutC but not GST alone (Fig. S1). Mass spectrometry analysis revealed that these bands correspond to the secretin OutD. This result suggests that OutC and OutD interact within the functional T2SS.

\section{The $N$-domain of OutD interacts with two regions of OutC}

To test if OutC and OutD interact in the absence of other components of the T2SS, in vitro pull-down assays were employed. OutD $\mathrm{D}_{28-710}$ was produced in E. coli $\mathrm{C} 41(\mathrm{DE} 3) / \mathrm{pLysS}$ and purified with Ni-NTA agarose. As with all OutD constructs used here, the signal sequence was deleted and a His tag was fused to the C-terminus (Fig. 1). GST-OutC was expressed in E. coli BL21 (DE3) and immobilized on Glutathione Sepharose. OutD $28-710$ bound to GSTOutC but not to GST alone (Fig. 2A). To map this interaction, a series of GST-OutC truncations were used. OutD $\mathrm{D}_{28-710}$ bound to all the fusions tested except $\mathrm{OutC}_{161-272}$, consisting of the PDZ domain only (Fig. 2A, lane 5). This reveals that OutD does not interact with the PDZ domain. OutD $\mathrm{D}_{28-710}$ did however bind to the periplasmic region of OutC corresponding approximately to the HR region (residues 40 to 172) (Fig. 2A, lane 6). In addition, a larger amount of OutD $\mathrm{D}_{28-710}$ bound to fusions including the TMS (Fig. 2A, lanes 2, 7 and 8). Since deletions within the two latter fusions $\left(\mathrm{OutC}_{\Delta 103-172}\right.$ and $\left.\mathrm{OutC}_{\Delta 41-127}\right)$ fully cover the HR (Fig. 
1 1), OutD may also bind to the remaining region of OutC (residues 1 to 40), which carries the

2 TMS. These results suggest that OutD may interact with two distinct regions of OutC.

To dissect these putative interacting sites within OutC, we used OutD derivatives consisting of the N-terminal region, which apparently protrudes into the periplasm (Chami et al., 2005). In pull-down assay, OutD $28-357$ interacted with the $\mathrm{N}$-terminal region of OutC, carrying the TMS, OutC $1-42$ (Fig. 2B, lane 3). Since, OutD ${ }_{28-357}$ did not bind to GST-OutM $2-46$, which comprises the TMS of OutM (Fig. 2B, lane 4), it is plausible that OutD interacts specifically with the TMS of OutC or region proximal to the TMS. When we probed the periplasmic region of OutC, OutD ${ }_{28-285}$ bound to $\mathrm{OutC}_{128-272}$, but not to $\mathrm{OutC}_{161-272}$ (Fig. $2 \mathrm{C}$, lanes 1 and 6). This binding site can therefore be mapped to the region between residues 128 to 160 of OutC. Since OutD $\mathrm{D}_{28-285}$ bound to OutC $_{60-99 / 124-272}$, but not to OutC $40-102 / 173-272$, (Fig. $1 \mathrm{~A}$ and $2 \mathrm{C}$, lanes 5 and 3), no other interacting sites could be detected within the periplasmic region of OutC from residue 40 to 272.

NMR spectroscopy reveals an interaction between the non-PDZ periplasmic region of OutC and the N-domain of OutD.

NMR spectroscopy was used to investigate the interaction between the periplasmic regions of OutC and OutD in solution. Two constructs of OutC were used, the first $\left(\mathrm{OutC}_{60-272}\right)$ comprises the periplasmic domain and the second $\left(\right.$ OutC $\left._{161-272}\right)$ corresponds to the PDZ domain only (Fig. 1). The 2D ${ }^{15} \mathrm{~N}-\mathrm{HSQC}$ spectra of the PDZ domain (Fig. 3A spectra in red) reveals well dispersed peaks which do not change in position when compared with the 2D ${ }^{15} \mathrm{~N}-\mathrm{HSQC}$ spectra of the entire periplasmic region of OutC (Fig. 3A spectra in green). This result suggests that the PDZ domain makes no or very few contacts with other domains present in the periplasmic region of OutC and is consistent with a "beads on a string model" of the domain architecture of the periplasmic region of OutC in the absence of other proteins. 
1 The two spectra were then recorded again but this time in the presence of unlabelled OutD $\mathrm{D}_{28-}$ 2285 at a molar ratio of $2: 1(0.5 \mathrm{mM}$ PDZ domain or $0.5 \mathrm{mM}$ periplasmic region of OutC to 0.25 $3 \mathrm{mM}$ OutD $28-285)$. The signal from the PDZ domain is unaffected by the presence of OutD

4 (compare the almost identical red spectra in Fig. 3A and B) showing that it is not involved in 5 an interaction with OutD. The observed major loss of intensity of the peaks from the non-PDZ 6 region of OutC (the signal strength of the green spectra in Fig. 3B is reduced compared to Fig. $7 \quad 3 \mathrm{~A}$ but only significantly for the non-PDZ peaks) indicates that it is the non-PDZ region 8 (mainly corresponding to HR) that interacts with the N-domain of OutD forming a complex 9 which leads to peak broadening and intensity loss. The intensity loss of the non-PDZ peaks may be best appreciated by inspection on Fig. 3C which shows the effect of adding OutD 11 (green) compared to OutC in the absence of OutD (red).

\section{A short region of OutC interacts with the two distinct sites within the N-domain of OutD}

To map the interaction site(s) within OutD, we divided the N-domain into two derivatives, OutD $\mathrm{D}_{28-112}$ and $\mathrm{OutD}_{116-285}$, one carrying the subdomain $\mathrm{N} 0$ and the other comprising the subdomains N1, N2 and the beginning of N3 (Fig. 1B). In co-purification assays, both derivatives behaved like the entire $\mathrm{N}$-domain $\left(\mathrm{OutD}{ }_{28-285}\right)$ being co-purified on GST-OutC $_{60-272}$, but not on GST-OutC $161-272$ (Fig. 4, lanes 1, 5 and 6 and Fig. S2). This suggests that two distinct sites of OutD, one located in the NO subdomain and another located within the N1-N2-N3' subdomains of OutD, interact with the periplasmic region of OutC. To

21 focus in on the second binding site, the N1 subdomain was removed from OutD $116-285$ (Fig. 4, lane 7). This did not apparently affect the binding of the resulting construct OutD $_{192-285}$. Conversely, removing of the C-terminal half of N2 and the beginning of N3 prevented the binding of OutD $\mathrm{D}_{116-221}$ to GST-OutC $60-272$ (Fig. 4, lane 8). This suggests that the second binding site of OutD involves residues 222 to 285 . 
Next, we examined, if these two putative binding sites of OutD interact with the two

2 putative binding sites identified within OutC. In pull-down assays, GST-OutC $1-42$ bound

3 OutD $_{28-154}$ but not OutD ${ }_{116-285}$ (not shown), suggesting that the $\mathrm{N}$-terminal region of OutC

4 interacts with the N0 subdomain of OutD. Conversely, both OutD $\mathrm{D}_{28-112}$ and OutD $116-285$ bound 5 specifically to GST-OutC ${ }_{139-272}$ but not to GST-OutC ${ }_{161-272}$ (Fig. 5A and 5B lanes 1 and 2).

6 This suggests that the two distinct regions of the N-domain of OutD interact with the same 7 segment of OutC, comprising residues 139 to 160 . This segment was named OutCsip (secretin $8 \quad$ interacting peptide).

9

\section{The two binding sites of the $N$-domain of OutD recognise different regions of OutCsip}

We next explored whether the OutCsip peptide alone can interact with OutD. Three peptides, OutC $_{131-158}$, OutC $139-162$ and OutC $_{144-158}$ (Fig. S3), were fused to GST and probed in copurification assays with $\operatorname{OutD}_{28-112}$ and $\mathrm{OutD}_{116-285}$. OutD $\mathrm{D}_{28-112}$ bound to all these peptides (Fig. 5A, lanes 3, 5 and 6). Conversely, OutD ${ }_{116-285}$ did not bind to the shorter peptide, OutC$_{144-158}$, whereas it bound to OutC $\mathrm{C}_{131-158}$ and $\mathrm{OutC}_{139-162}$ (Fig. 5B, lanes 3, 5 and 6). These results show that the two distinct sites of OutD interact in vitro with the 20 residue stretch of OutC (residues 139 to 158 ). Moreover, the binding site of OutD $_{28-112}$ (the N0 subdomain) can be mapped to a 15 residue peptide (residues 144 to 158 ).

To further compare the binding of OutCsip to the two sites of OutD, the three proteins were co-expressed from the tac promoter of pGEX-6P-3 in the order GST-OutC $128-272$,

21 OutD $_{28-112}$ and OutD $_{116-285}$. The ratio between the three proteins was similar to that in the pairwise co-expressions (Fig. 5C). In co-purification assay with the three proteins, a lower amount of OutD $\mathrm{D}_{28-112}$ was bound, in comparison with that observed with pairwise coexpressed GST-OutC $128-272$ and $\mathrm{OutD}_{28-112}$ (Fig. 5C, lanes 1 and 3), indicating that OutD $28-112$ 
1285 bound to OutC $\mathrm{C}_{128-272}$ was two-fold higher than that observed with pairwise co-expressed

2 GST-OutC ${ }_{128-272}$ and OutD $_{116-285}$ (Fig. 5C, lanes 1 and 2). This indicates that OutD $_{28-112}$

3 enhances the binding of OutD ${ }_{116-285}$ to OutCsip.

4

A single amino acid substitution in the OutCsip region blocks secretion in E. chrysanthemi

6 To examine the functional relevance of the OutCsip region, two C-terminal truncations of

7 OutC were generated, one at the beginning of OutCsip $\left(\operatorname{OutC}_{1-141}\right)$ and another just after it

$8 \quad\left(\right.$ OutC $\left.\mathrm{C}_{1-159}\right)$ (Fig. S3B). OutC $\mathrm{C}_{1-159}$ restored the secretion of PemA in a complementation test

9 with E. chrysanthemi $\triangle$ outC strain (Fig. 6B). In contrast, OutC ${ }_{1-141}$ was non-functional,

10 indicating that OutCsip is essential for the function of the protein. We further substituted

11 several residues in the OutCsip region, namely V143A, V144A, G149A, E152A, Y157A,

12 S137D, R142I and R150L. Immunoblotting with OutC-antibodies revealed all the mutant

13 proteins except OutC ${ }^{\mathrm{G} 149 \mathrm{~A}}$ once expressed in E. chrysanthemi $\Delta$ outC strain (Fig. 6A).

14 OutC $^{\mathrm{G} 149 \mathrm{~A}}$ was not detected and probably degraded. In a complementation test, all the mutant

15 proteins, except OutC ${ }^{\mathrm{G} 149 \mathrm{~A}}$, fully restored secretion of the pectinases to the wild type level

16 (Fig. 6B). In addition, OutC ${ }^{\mathrm{V} 143 \mathrm{~A}}$, whose quantity was not apparently affected, only partially

17 restored secretion of pectinases (Fig. 6). Among three putative $\beta$ strands predicted in OutCsip

18 by Jpred algorithm (Cole et al., 2008), one overlaps Val143 (Fig. S3). Thus, Val143 was

19 replaced by serine, which is considered to be less compatible with such a secondary structure

20 (Chou and Fasman, 1978). The quantity of OutC ${ }^{\mathrm{V} 143 \mathrm{~S}}$ was not affected (Fig. 6A), but no

21 pectinase was secreted by A3618 expressing this mutant protein (Fig. 6B).

The substitution V143S prevents interaction of OutCsip with OutD ${ }_{116-285}$

24 To examine the effect of the V143S mutation on the OutC-OutD interaction, this substitution

25 was introduced into several GST-OutC derivatives. GST-OutC ${ }_{128-272}{ }^{\text {V143S }}$ was completely 
1 degraded (not shown), indicating a strong deleterious effect of this mutation on protein 2 stability. In contrast, GST-OutC ${ }_{131-158^{\mathrm{V} 143 \mathrm{~S}}}$ was stable (Fig. 5A and 5B, lane 4). In co3 purification assays, a similar amount of OutD $_{28-112}$ was bound to GST-OutC $131-158$ and to GST4 OutC $_{131-158}{ }^{\text {V143S }}$ (Fig. 5A, lanes 4 and 5). Conversely, OutD ${ }_{116-285}$ was not bound to the mutant 5 fusion protein (Fig. 5B and S3). Thus, the V143S substitution affects the interaction of 6 OutC $_{131-158}$ with only one interaction site of OutD located in N1-N3' subdomains.

7

\section{Discussion}

In this article, we provide strong evidence that the secretin OutD interacts with OutC, an inner membrane component of the T2SS. We discovered that the N-terminal (periplasmic) domain of OutD interacts with the short segment of OutC (residues 139 to 158), named OutCsip (secretin interacting peptide). OutCsip is proximal to the PDZ domain and is located at the Cterminus of the so-called homology region (HR) (Gérard-Vincent et al., 2002). The PDZ of OutC (residues 175 to 272) plays a specific role and is necessary for the secretion of pectate lyases, although it is fully dispensable for the secretion of certain other proteins (Bouley et al., 2001). The short segment located between OutCsip and PDZ appears to be not essential, since OutC $_{\Delta 163-169}$ is secretion competent (Fig. S3). In contrast, the OutCsip region is indispensable for the function of OutC. Indeed, OutC $_{1-159}$ possessing this region allows for the secretion of PemA, but OutC ${ }_{1-141}$, lacking this peptide, does not.

Site directed mutagenesis of the OutCsip region reveals that the V143S substitution fully blocks secretion while the V143A substitution only partially affects it. Val143 of OutC may be directly involved in a hydrophobic interaction with OutD which is partly mimicked by the Ala substitution but not by the Ser substitution. Indeed, V143S mutation prevents interaction of the OutC $\mathrm{C}_{131-159}$ peptide with $\mathrm{OutD}_{116-285}$, the $\mathrm{N} 1-\mathrm{N} 3$ ' region of OutD. Alternatively, Val143 may promote a conformation of OutC needed for binding, a 
1 conformation that is significantly distorted by the Ser substitution. Introduction of the V143S

2 substitution in GST-OutC $128-272$ provokes degradation of the protein consistent with increased

3 flexibility and susceptibility to cleavage. The functional relevance of OutCsip was further

4 shown by interference of this peptide with the pectinase secretion (Fig. S5). A similar

5 phenomenon has been observed by expression of the entire periplasmic domain of the $P$.

6 aeruginosa GspC (Bleves et al., 1999).

We showed that the OutCsip peptide interacts in vitro with two distinct regions of OutD: one, located in the N0 subdomain and another, involving the N2 and N3' subdomains.

Our results suggest that these two binding sites of OutD, (OutD $28-112$ and OutD $_{116-285}$ respectively) either interact with two overlapping subsites within OutCsip, or have different modes of binding to the same binding site. Indeed, both OutD $\mathrm{D}_{28-112}$ and $\operatorname{OutD}_{116-285}$ bind to OutC $_{131-158}$ and OutC $\mathrm{C}_{139-162}$, but only OutD $\mathrm{D}_{28-112}$ binds to OutC ${ }_{131-158}{ }^{\mathrm{V}_{143 \mathrm{~S}}}$ and to OutC $\mathrm{C}_{144-158}$ (Fig. S3C). These data suggest that the minimal binding site of $\operatorname{OutD}_{28-112}$ but not of OutD $\mathrm{D}_{116-}$ 285 can be mapped within a 15 residue stretch (residues 144 to 158). The three-partner binding experiments showed two phenomena indicative of different binding modes of OutD $\mathrm{D}_{28-112}$ and OutD ${ }_{116-285}$ to OutCsip: i) a lower binding of OutD $28-112$ in the presence of OutD $_{116-285}$ that suggests a competition between $\operatorname{OutD}_{28-112}$ and OutD ${ }_{116-285}$ for binding to OutCsip and ii) a higher binding of OutD $\mathrm{D}_{116-285}$ in the presence of OutD $\mathrm{D}_{28-112}$ that suggests a synergistic binding of OutD $\mathrm{D}_{116-285}$ in the presence of OutD $28-112$ (Fig. $5 \mathrm{C}$ ).

Dissection of OutC showed that, in addition to OutCsip, another site, which involves the TMS of OutC and/or the proximal region (residues 1 to 42), interacts in vitro with the Ndomain of OutD. Detailed mapping of the interacting sites was compromised by a low solubility of GST-OutC ${ }_{1-42}$ when it was co-expressed with the tested OutD derivatives (not shown). However, pull-down assay indicates that the NO subdomain of OutD is involved in this interaction. It seems unlikely that the soluble folded N0 subdomain of OutD comes into 
1 contact with the TMS of OutC buried in the inner membrane. The second binding site of

2 OutC is likely to be in the periplasmic region close to the TMS. Therefore, the N0 subdomain

3 of OutD interacts in vitro with two distinct regions of OutC, while the OutCsip segment

4 interacts with two distinct regions of OutD.

In various T2SSs, the genes encoding GspC and GspD are located together as part of

6 the same operon (Cianciotto, 2005; Evans et al., 2008). One reason for such a gene

7 organization may be that $\mathrm{GspC}$ and $\mathrm{GspD}$ act in partnership. The two proteins can only be exchanged pair-wise between evolutionary distant T2SSs (de Groot et al., 2001; Michel et al., 2007). This implies the presence of species-specific GspC-GspD interactions necessary for the correct functioning of the protein pair. The interactions between OutC and OutD we discovered could be a part of these interactions.

Formation of a tight complex between the periplasmic regions of OutC and OutD in vitro may reflect the formation of a similar structure within the functional T2SS. Indeed, OutD was efficiently co-purified with GST-OutC from the E. chrysanthemi secretion system (Fig. S1). The cylindrical dodecameric structure formed by the N-terminal domain of the secretin apparently protrudes into the periplasm (Chami et al., 2005, Korotkov et al., 2009) and, hence, it can easily interact with the periplasmic region of OutC. The stoichiometry of the OutC-OutD complex within the T2SS remains unknown. In contrast to PulD and many other secretins, OutD does not form SDS-resistent multimers when it is expressed at physiological level (Guilvout et al., 1999). Therefore, it was not possible to estimate the multimeric state of the full-length OutD co-purified with GST-OutC from the E. chrysanthemi T2SS. Nevertheless, the derivatives consisting of the periplasmic regions of OutC and OutD were co-eluted in an equimolar ratio from Glutathione Sepharose (Fig. S4A), suggesting a dodecameric stoichiometry of OutC in vivo. Size exclusion chromatography did not allow the exact stoichiometry of these complexes to be determined, since once released from GST, they 
1 were unstable (Fig. S4B). Together, OutD and OutC may take the shape of a flexible cage-

2 like structure spanning the periplasm and stabilizing the complex across the two membranes,

3 reminiscent of a model suggested by Filloux et al. (1998). The periplasmic regions of the

4 secretin and of OutC possess several disordered regions, probably acting as flexible linkers

5 (Korotkov et al., 2006, Korotkov et al., 2009). This structural flexibility can facilitate their

6 interactions with protein partners during secretion. Since both OutC and OutD are involved in

7 the recognition of secreted proteins (Lindeberg et al., 1996; Shevchik et al., 1997; Bouley et

8 al., 2001), it is plausible that the interaction between OutC and OutD would modulate their

9 affinity for exoproteins. We found that two distinct regions of OutD interact either with the

10 same site or with two partially overlapping sites located within the OutCsip segment.

11 Moreover, the binding of one interacting region $\left(\mathrm{OutD}_{28-112}\right)$ to OutCsip increases the binding

12 of the other interacting region $\left(\right.$ OutD $\left._{116-285}\right)$. Conversely, the binding of OutD $116-285$ to OutCsip

13 reduces the binding of $\mathrm{OutD}_{28-112}$ to OutCsip. Considering that in vitro OutD $28-154$ interacts

14 also with the region proximal to the TMS of OutC, we can suggest a functional implication of

15 these phenomena. In the course of secretion, the N0 subdomain of OutD (OutD $\left.\mathrm{D}_{28-112}\right)$ could

16 interact with the OutCsip region. This interaction increases affinity of OutCsip to the other

17 interaction site located in OutD ${ }_{116-285}$ and promotes its binding to OutCsip. Subsequently, the

binding of OutD $\mathrm{D}_{116-285}$ to OutCsip provokes the dissociation of N0 from OutCsip and its

binding to the region proximal to the TMS of OutC. Therefore, in the course of secretion, the

OutCsip peptide could move between the two interaction sites of the N-domain of OutD and,

21 thus, switch the T2SS machinery between two functional states. Hence, OutC and OutD can

22 act together as an allosterically cooperative system (Whitty, 2008); the binding of a protein

23 partner to one site alters the binding affinity of another site on the protein. Future studies will

24 elucidate the detailed molecular architectures and residues involved in these interactions. 


\section{Experimental procedures}

2 Bacterial strains and growth conditions

3 The bacterial strains and plasmids used in this study are listed in Table S1. The bacteria were

4 usually grown in Luria-Bertani (LB) or M9 minimal medium at $30^{\circ} \mathrm{C}$ with shaking at 150

$5 \mathrm{rpm}$. If necessary, antibiotics were added at the following final concentrations: ampicillin, 150

$6 \mu \mathrm{g} \mathrm{ml}^{-1}$; kanamycin, $100 \mu \mathrm{g} \mathrm{ml}^{-1}$ and chloramphenocol, $50 \mu \mathrm{g} \mathrm{ml}^{-1}$.

7

\section{Plasmid construction}

9 DNA cloning and manipulations were performed using standard methods. Site directed 10 mutagenesis was performed using the QuickChange kit (Stratagene). The primers used in this 11 study are listed in Table S2. The nucleotide sequences of mutant and amplified genes were checked (Cogenics). To generate plasmids expressing GST-OutC derivatives, corresponding gene fragments were amplified by PCR and subcloned into pGEX-6P-3 in frame with the

14 GST-encoding sequence. Since the beginning of the outD gene overlaps the end of outC, an 15 Amber stop-codon was created at the beginning of outD by the introduction of an XbaI site.

16 To generate plasmids expressing the signal sequence-less OutD $\mathrm{His}_{\text {His }}$ derivatives, corresponding 17 gene fragments were amplified by PCR and subcloned into the NdeI/HindII sites of pET20b 18 in frame with a 6His-encoding sequence. To generate plasmids co-expressing GST-OutC and 19 OutD $_{\text {His }}$ derivatives, corresponding outD gene fragments preceded by an RBS were subcloned 20 from an appropriate pET20b(+) construct into XbaI/SspI sites of pGEX-6P-3 downstream of 21 the GST-outC sequence. More details are described in supplementary information.

Protein purification and analysis

${ }^{15} \mathrm{~N}$-labelled OutC derivatives fused to GST were produced in E. coli BL21(DE3) carrying a corresponding pGX-o $\mathrm{C}_{\mathrm{X}-\mathrm{Y}}$ plasmid ( $\mathrm{oC}_{\mathrm{X}-\mathrm{Y}}$ for any OutC derivative, as in Table $\mathrm{S} 1$ ). Bacteria 
1 were grown in M9 medium supplemented with glucose, $10 \mathrm{~g} \mathrm{l}^{-1}$, and ${ }^{15} \mathrm{NH}_{4} \mathrm{Cl}, 1 \mathrm{~g} \mathrm{l}^{-1}$. At an

$2 \mathrm{OD}_{600}$ of 0.7 , isopropyl- $\beta$-D-thiogalactopyranoside (IPTG) was added to $1 \mathrm{mM}$, and the

3 cultures were grown for an additional $5 \mathrm{~h}$. The cells were broken with a French press and the

4 protein was purified on Glutathione Sepharose as described previously (Login and Shevchik,

5 2006). Protein was dialysed against $20 \mathrm{mM}$ Tris- $\mathrm{HCl} \mathrm{pH} \mathrm{9,} 1 \mathrm{mM}$ EDTA and loaded onto a

6 Hi Trap Q-FF column (GE Healthcare) equilibrated with the same buffer. The pure protein

7 was eluted by $\mathrm{NaCl}$ gradient, concentrated and washed in $10 \mathrm{mM}$ Tris- $\mathrm{HCl} \mathrm{pH} 7,0.1 \mathrm{mM}$

8 EDTA with centricon YM-10 or YM-3 (Millipore).

OutD $_{\text {His }}$ derivatives used in pull down assays were produced in E. coli $\mathrm{C} 41(\mathrm{DE} 3) / \mathrm{pLys}$

10 cells carrying a corresponding $\mathrm{pET}-\mathrm{oD}_{\mathrm{X}-\mathrm{Y}}$ plasmid $\left(\mathrm{oD}_{\mathrm{X}-\mathrm{Y}}\right.$ for any OutD derivative, as in

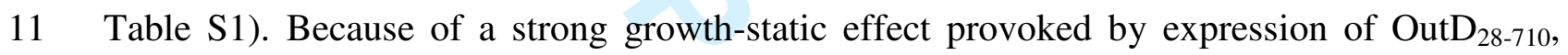

12 induction time was limited to $45 \mathrm{~min}$. The proteins were purified using Ni-NTA agarose, as

13 described (Login and Shevchik, 2006). The recombinant proteins were solubilized in $100 \mathrm{mM}$

14 sodium phosphate, $10 \mathrm{mM}$ Tris- $\mathrm{HCl}, 100 \mathrm{mM} \mathrm{NaCl} \mathrm{pH} \mathrm{8.0,} \mathrm{then} \mathrm{bound} \mathrm{to} \mathrm{the} \mathrm{resin,} \mathrm{washed}$

15 with the same buffer at $\mathrm{pH} 6.8$ and eluted with a linear gradient of histidine. Purification of

16 OutD $28-710$ was performed in the presence of $1 \%$ Triton $\mathrm{X}-100$. For NMR experiments, an

17 additional purification on a Hi Trap Q FF column (GE Healthcare) was performed as above.

SDS-PAGE and immunoblotting were performed as previously (Shevchik et al., 1997,

19 Bouley at al., 2001). Monoclonal tetra-His antibody (Qiagen), peroxidase conjugated Ni-NTA

20 (Qiagen) and anti-GST antibody (Sigma) were used as suggested by the manufacturers. 2D-

21 GE was performed as described by Kazemi-Pour et al. (2004).

To test the functionality of OutC mutant proteins, complementation assays were

23 performed with E. chrysanthemi A3618 $\Delta$ outC strain transformed with a pTdB-OC plasmid

24 carrying a corresponding mutant outC gene. Efficiency of secretion was estimated by 
1 immunoblotting of the secreted proteins in culture supernatants and whole cell extracts, as

2 described previously (Login and Shevchik, 2006).

3

Protein-protein interaction assays

In vitro GST pull-down assays were performed as described previously (Login and Shevchik, 2006). Equal amounts of GST or GST-OutC derivatives were immobilized on Glutathione Sepharose beads. The beads with bound GST-OutC derivatives have been systematically checked by SDS-PAGE prior to the binding assay and then diluted if necessary with noncharged resin to reach a similar amount of various fusion proteins. Next an equal amount of prey protein $\left(\right.$ OutD $D_{\text {His }}$ derivative) was loaded onto the resin with immobilized GST fusions,

11 incubated for $1 \mathrm{~h}$ and then washed. The bound proteins were eluted with Laemmli sample buffer, separated by SDS-PAGE and probed by immunoblotting with either OutD-antibodies, or Ni-NTA conjugated with peroxidase, or Tetra-His-antibodies (Quiagen). expressed in E. coli $\mathrm{BL} 21(\mathrm{DE} 3)$ carrying one of the pGX-oC $\mathrm{X}_{\mathrm{X}-\mathrm{Y}} \mathrm{o} \mathrm{D}_{\mathrm{X}-\mathrm{Y}}$ plasmids $\left(\mathrm{oC}_{\mathrm{X}-\mathrm{Y}}\right.$ and $\mathrm{oD}_{\mathrm{X}-\mathrm{Y}}$ for any OutC and OutD derivatives, as in Table S1). Bacteria were grown and induced with $1 \mathrm{mM}$ IPTG for $3 \mathrm{~h}$. Cells were resuspended in $50 \mathrm{mM}$ Tris- $\mathrm{HCl} \mathrm{pH} 8.0,100 \mathrm{mM} \mathrm{NaCl}$, 1 mM EDTA (Buffer B) supplemented with either $1 \%$ or $0.1 \%(\mathrm{v} / \mathrm{v})$ Triton X-100 and broken by sonication. Unbroken cells were eliminated at $6000 \mathrm{~g}$ for $10 \mathrm{~min}$ and the cell lysates were incubated with Glutathione Sepharose for $1 \mathrm{~h}$. Next, the resin was washed 3 times with buffer B supplemented with Triton X-100 and for an additional 3 times with buffer B without detergent. The bound proteins were eluted with Laemmli sample buffer, separated by SDSPAGE and probed by immunoblotting with either OutD-antibodies, or Ni-NTA conjugated with peroxidase, or Tetra-His-antibodies (Quiagen). 
In vivo co-purification of proteins bound to GST or GST-OutC during secretion was

2 performed from E. chrysanthemi A3618 cells carrying either pGEX-6P-3 or pTdB-GoC

3 plasmid. The bacteria were grown in $\mathrm{LB}$ to an $\mathrm{OD}_{600}$ of 0.7 and the proteins bound to GST or

4 GST-OutC were co-purified, as described above at the presence of $1 \%$ Triton X-100, and 5 analyzed by 2D-GE.

NMR experiments

NMR spectra were recorded at $303 \mathrm{~K}$ on a Bruker AvanceII spectrometer operating at a proton frequency of $600.13 \mathrm{MHz}$, equipped with a triple resonance $\mathrm{HCN}$ probe head and activelyshielded z-gradients. ${ }^{1} \mathrm{H}$ spectra were acquired using excitation sculpting with gradients for water suppression (Hwang and Shaka, 1995). ${ }^{1} \mathrm{H} /{ }^{15} \mathrm{~N}$ 2D correlation experiments (Palmer et al., 1991) were acquired via double INEPT transfer using sensitivity improvement. Phase sensitive detection in the indirect dimension used echo/antiecho-TPPI gradient selection, with decoupling during acquisition. Water was suppressed using a soft flip-back pulse. Data acquisition and processing were performed using the Bruker Topspin program. Chemical shifts were referenced to internal DSS (2,2-Dimethyl-2-silapentane-5-sulfonic acid) for ${ }^{1} \mathrm{H}$ directly and indirectly for ${ }^{15} \mathrm{~N}$ data. All samples for NMR spectroscopy were made up in 10 $\mathrm{mM}$ Tris/HCl, pH 7.0, $10 \mathrm{mM} \mathrm{CaCl} 2,10 \% \mathrm{D}_{2} \mathrm{O}, 100 \mu \mathrm{M}$ DSS. When recording $2 \mathrm{D}{ }^{15} \mathrm{~N}$ HSQC spectra, ${ }^{15} \mathrm{~N}$-labelled proteins were used at concentrations of 0.5 and $0.2 \mathrm{mM}$; unlabelled proteins were present at molar ratios of $2: 1$ and $1: 1(0.25 \mathrm{mM}$ and $0.2 \mathrm{mM}$, respectively). All spectra were kindly recorded by Dr H. Toms.

We are grateful to G. Condemine and other members of the Erwinia team for reading the manuscript, and for their valuable discussions. This work was supported by grants from the 
1 Centre National de la Recherche Scientifique (CNRS) and the Franco-British programme 2 Alliance 12109QK.

\section{References}

Bitter, W., Koster, M., Latijnhouwers, M., de Cock, H., and Tommassen, J. (1998) Formation of oligomeric rings by XcpQ and PilQ, which are involved in protein transport across the outer membrane of Pseudomonas aeruginosa. Mol Microbiol 27: 209-219.

Bleves, S., Lazdunski, A., and Filloux, A. (1996) Membrane topology of three Xcp proteins involved in exoprotein transport by Pseudomonas aeruginosa. J Bacteriol 178: 4297-4300.

Bleves, S., Gérard-Vincent, M., Lazdunski, A., and Filloux, A. (1999) Structure-function analysis of XcpP, a component involved in general secretory pathway-dependent protein secretion in Pseudomonas aeruginosa. J Bacteriol 181: 4012-4019.

Bouley, J., Condemine, G., and Shevchik, V.E. (2001) The PDZ domain of OutC and the Nterminal region of OutD determine the secretion specificity of the type II Out pathway of Erwinia chrysanthemi. J Mol Biol 308: 205-219.

Brok R., Van Gelder P., Winterhalter, M., Ziese, U., Koster, A.J., de Cock, H., et al. (1999) The C-terminal domain of the Pseudomonas secretin XcpQ forms oligomeric rings with pore activity. J Mol Biol 294: 1169-1179.

Burghout, P., Beckers, F., de Wit, E., van Boxtel, R., Cornelis, G.R., Tommassen, J., and Koster, M. (2004) Role of the pilot protein YscW in the biogenesis of the YscC secretin in Yersinia enterocolitica. J Bacteriol 186: 5366-5375.

Burghout, P., van Boxtel, R., Van Gelder, P., Ringler, P., Müller, S.A., Tommassen, J., and Koster, M. (2004) Structure and electrophysiological properties of the YscC secretin from the type III secretion system of Yersinia enterocolitica. J Bacteriol 186: 4645-4654. 
1 Chami, M., Guilvout, I., Gregorini, M., Rémigy, H., Müller, S.A., Valerio, M., et al. (2005)

2 Structural insights into the secretin PulD and its trypsin resistant core. J Biol Chem 280: $3 \quad 37732-37741$.

4 Cianciotto, N.P. (2005) Type II secretion: a protein secretion system for all seasons. Trends 5 Microbiol 13: 581-588.

Chou, P.Y., and Fasman, G.D. (1978) Empirical predictions of protein conformation. Annu Rev Biochem 47: 251-276.

Cole, C., Barber, J.D., and Barton, G.J. (2008) The Jpred 3 secondary structure prediction server. Nucleic Acids Res 36: W197-W201.

Condemine, G., and Shevchik, V.E. (2000) Overproduction of the secretin OutD suppresses the secretion defect of an Erwinia chrysanthemi outB mutant. Microbiology 146: 639-647.

Daefler, S., Russel, M. and Model, P. (1997) Module swaps between related translocator proteins $\mathrm{pIV(f1),pIV(IKe)}$ and PulD: identification of a specificity domain. $J$ Mol Biol 266: 978-992.

de Groot, A., Koster, M., Gérard-Vincent, M., Gerritse, G., Lazdunski, A., Tommassen, J., and Filloux, A. (2001) Exchange of Xcp (Gsp) secretion machineries between Pseudomonas aeruginosa and Pseudomonas alcaligenes: species specificity unrelated to substrate recognition. J Bacteriol 183: 959-967.

Douet, V., Loiseau, L., Barras, F., and Py, B. (2004) Systematic analysis, by the yeast twohybrid, of protein interaction between components of the type II secretory machinery of Erwinia chrysanthemi. Res Microbiol 155: 71-75.

Evans, F.F., Egan, S., and Kjelleberg, S. (2008) Ecology of type II secretion in marine gammaproteobacteria. Environ Microbiol 10: 1101-1107.

24 Ferrandez, Y, Condemine, G. (2008) Novel mechanism of outer membrane targeting of proteins in Gram-negative bacteria. Mol Microbiol 69: 1349-1357. 
1 Filloux, A. (2004) The underlying mechanisms of type II protein secretion. Biochim Biophys $2 \quad$ Acta 1694: 163-179.

3 Genin, B., and Boucher, C.A. (1994) A superfamily of proteins involved in different secretion 4 pathways in gram-negative bacteria: modular structure and specificity of the N-terminal $5 \quad$ domain. Mol Gen Genet 243: 112-118.

6 Gérard-Vincent, M., Robert, V., Ball, G., Bleves, S., Michel, G.P., Lazdunski, A., and Filloux, A. (2002) Identification of XcpP domains that confer functionality and specificity to the Pseudomonas aeruginosa type II secretion apparatus. Mol Microbiol 44: 1651-1665.

Guilvout, I., Hardie, K.R., Sauvonnet, N., Pugsley, A.P. (1999) Genetic dissection of the outer membrane secretin PulD: are there distinct domains for multimerization and secretion specificity? J Bacteriol 181: 7212-7220.

Guilvout, I., Chami, M., Engel, A., Pugsley, A.P., and Bayan, N. (2006) Bacterial outer membrane secretin PulD assembles and inserts into the inner membrane in the absence of its pilotin. EMBO J 25: 5241-5249.

Guilvout, I., Chami, M., Berrier, C., Ghazi, A., Engel, A., Pugsley, A.P., and Bayan, N. (2008) In vitro multimerization and membrane insertion of bacterial outer membrane secretin PulD. J Mol Biol 382: 13-23.

Hardie, K.R., Lory, S., and Pugsley, A.P. (1996) Insertion of an outer membrane protein in Escherichia coli requires a chaperone-like protein. EMBO J 15: 978-988.

Hwang, T.-L., and Shaka, A.J. (1995) Water suppression that works: excitation sculpting using arbitrary waveforms and pulse field gradients. J Magn Reson 112: 275-279.

Kazemi-Pour, N., Condemine, G., and Hugouvieux-Cotte-Pattat, N. (2004) The secretome of the plant pathogenic bacterium Erwinia chrysanthemi. Proteomics 4: 3177-3186. 
1 Korotkov, K.V., Krumm, B., Bagdasarian, M., and Hol, W.G. (2006) Structural and

2 functional studies of EpsC, a crucial component of the type 2 secretion system from Vibrio $3 \quad$ cholerae. J Mol Biol 363: 311-321.

4 Korotkov, K.V., Pardon, E., Steyaert, J., and Hol, W.G. (2009) Crystal structure of the N5 terminal domain of the secretin GspD from ETEC determined with the assistance of a $6 \quad$ nanobody. Structure 17: 255-265.

7 Lee, H.M., Chen, J.R., Lee, H.L., Leu, W.M., Chen, L. Y., and Hu, N.T. (2004) Functional dissection of the XpsN (GspC) protein of the Xanthomonas campestris pv. campestris type II secretion machinery. J Bacteriol 186: 2946-2955.

Lee, M.S., Chen, L.Y., Leu, W.M., Shiau, R.J., and Hu, N.T. (2005) Associations of the major 11 pseudopilin XpsG with $\mathrm{XpsN}(\mathrm{GspC})$ and secretin XpsD of Xanthomonas campestris pv. campestris type II secretion apparatus revealed by cross-linking analysis. J Biol Chem 280: 4585-4591.

Lee, H.M., Wang, K.C., Liu, Y.L., Yew, H.Y., Chen, L.Y., Leu, W.M., et al. (2000) Association of the cytoplasmic membrane protein $\mathrm{XpsN}$ with the outer membrane protein $\mathrm{XpsD}$ in the type II protein secretion apparatus of Xanthomonas campestris pv. campestris. J Bacteriol 182: 1549-1557.

Lindeberg, M., Salmond, G.P.C., and Collmer, A. (1996) Complementation of deletion mutants in a cloned functional cluster of Erwinia chrysanthemi out genes with Erwinia carotovora out homologues reveals OutC and OutD as candidate gatekeepers of speciesspecific secretion of proteins via the type II pathway. Mol Microbiol 20: 175-190.

Linderoth, N.A., Simon, M.N., and Russel, M. (1997) The filamentous phage pIV multimer visualized by scanning transmission electron microscopy. Science 278: 1635-1638. 
1 Login, F.H., and Shevchik, V.E. (2006) The single transmembrane segment drives self-

2 assembly of OutC and the formation of a functional type II secretion system in Erwinia 3 chrysanthemi. J Biol Chem 281: 33152-33162.

4 Michel, G.P., Durand, E., and Filloux, A. (2007) XphA/XqhA, a novel GspCD subunit for $5 \quad$ type II secretion in Pseudomonas aeruginosa. J Bacteriol 189: 3776-3783.

6 Nouwen, N., Stahlberg, H., Pugsley, A.P., and Engel, A. (2000) Domain structure of secretin $7 \quad$ PulD revealed by limited proteolysis and electron microscopy. EMBO J 19: 2229-2236.

8 Palmer, A.G., Cavanagh, J., Wright, P.E. and Rance, M. (1991) Sensitivity improvement in 9 proton-detected two-dimensional heteronuclear correlation NMR spectroscopy. J Magn Reson 93: 151-170.

11 Possot, O.M., Gérard-Vincent, M., and Pugsley, A.P. (1999) Membrane association and 12 multimerization of secreton component PulC. J Bacteriol 181: 4004-4011.

13 Possot, O.M., Vignon, G., Bomchil, N., Ebel, F., and Pugsley, A.P. (2000) Multiple 14 interactions between pullulanase secreton components involved in stabilization and 15 cytoplasmic membrane association of PulE. J Bacteriol 182: 2142-2152.

16 Pugsley, A.P., Poquet, I., and Kornacker, M.G. (1991) Two distinct steps in pullulanase 17 secretion by Escherichia coli K12. Mol Microbiol 5: 865-873.

18 Shevchik, V.E., Robert-Baudouy, J., and Condemine, G. (1997) Specific interaction between 19 OutD, an Erwinia chrysanthemi outer membrane protein of the general secretory pathway, 20 and secreted proteins. EMBO J 16: 3007-3016.

21 Shevchik, V.E., and Condemine, G. (1998) Functional characterization of the Erwinia 22 chrysanthemi OutS protein, an element of a type II secretion system. Microbiology 144: 3219-3228. 
1 Thomas, J.D., Reeves, P.J., and Salmond, G.P. (1997) The general secretion pathway of 2 Erwinia carotovora subsp. carotovora: analysis of the membrane topology of OutC and 3 OutF. Microbiology 143: 713-720.

4 Voulhoux, R., Ball, G., Ize, B., Vasil, M.L., Lazdunski, A., Wu, L.F., and Filloux, A. (2001) 5 Involvement of the twin-arginine translocation system in protein secretion via the type II 6 pathway. EMBO J 20: 6735-6741.

7 Whitty, A. (2008) Cooperativity and biological complexity Nat Chem Biol 4: 435-439.

$9 \quad$ Figure legends

Fig. 1. Schematic diagrams of OutC (A) and OutD (B) and their truncated derivatives used in the study.

13 The position of HR (homology region) is according to Gérard-Vincent et al. (2002). The subdomain structure of the N-terminal region of OutD (subdomains N0 to N3) is according to the 3D structure of peri-GspD (Korotkov et al., 2009): 45\% of amino acid identity among 235 residues with OutD. L1 to L3 indicate disordered linker regions; SP, signal peptide; S, S-

17 domain; and TMS, transmembrane segment.

18 All the OutD derivatives used in the study possess a C-terminal His tag.

$19 *$ The sequences of the OutCsip derivatives are shown in Fig. S3.

21 Fig. 2. Mapping of the OutC regions interacting with OutD.

22 A. OutD interacts with two distinct regions of OutC. Equal amounts of GST-OutC derivatives 23 (indicated on top) were immobilized on Glutathione Sepharose beads, as checked by SDS24 PAGE prior to the binding assay. Next, an equal amount of OutD $28-710$ was loaded onto these 25 matrices, incubated for $1 \mathrm{~h}$ and washed. Bound proteins were eluted with Laemmli sample 
1 buffer, separated by SDS-PAGE and either stained (upper panels) or probed with OutD-

2 antibodies (lower panels). GST-fused proteins are indicated by asterisks and OutD derivatives

3 by triangles. Lower species, reacting with GST-antibodies, are GST-OutC degradation

4 products generated during the incubation. Amounts of bound $\operatorname{OutD}_{28-710}$ were estimated by

5 densitometry (indicated as a percentage of that to GST-OutC on bottom of the left panel).

6 B. N-domain of OutD interacts with the N-terminal region of OutC. Pull-down experiments 7 were performed as in (A). OutD $28-357$ was used in place of OutD $28-710$.

8 C. N-domain of OutD interacts with the HR region of OutC. GST-OutC derivatives (indicated on top) and OutD $28-285$ were co-expressed in E. coli BL21(DE3) from the single plasmids and then used in co-purification assay on Glutathione Sepharose.

\section{Fig. 3. 2D ${ }^{15} \mathrm{~N}-\mathrm{HSQC}$ NMR analysis of the interaction of OutC and OutD.}

A and B. $2 \mathrm{D}{ }^{15} \mathrm{~N}$-HSQC spectrum of the ${ }^{15} \mathrm{~N}$-labelled periplasmic region of OutC $\left(\mathrm{OutC}_{60-272}\right.$ spectra in green) overlaid with the $2 \mathrm{D}{ }^{15} \mathrm{~N}$-HSQC spectrum of the ${ }^{15} \mathrm{~N}$-labelled PDZ domain (OutC ${ }_{161-272}$ spectra in red). In panel (A) the spectra are recorded in the absence of OutD and in panel (B) in the presence of $0.25 \mathrm{mM}$ OutD (unlabelled OutD ${ }_{28-285}$ ). The concentration of the OutC derived proteins was $0.50 \mathrm{mM}$. The PDZ domain spectra (red) in panel A is almost identical to that in panel B showing there is no interaction with OutD. The loss of intensity for the non-PDZ region (HR) of the spectra in panel B compared to panel A (green spectra) shows it is the HR region that interacts with OutD.

C. $\mathrm{S}$ proximate uperimposition of the ${ }^{15} \mathrm{~N}-\mathrm{HSQC}$ spectra for OutC $\mathrm{C}_{60-272}$ in the absence (red) and presence (green) of unlabelled OutD $\mathrm{D}_{28-285}$. The broadening of some of the peaks in the presence of OutD (green) is clear and confirms the interaction between OutC and OutD. The broadened peaks are not those assigned to the PDZ domain and hence the interaction between

OutC and OutD does not involve the PDZ domain. 
2 Fig. 4. Mapping of the OutD regions interacting with the periplasmic region of OutC.

3 A. Whole cell extracts of $E$. coli BL21(DE3) co-expressing GST-OutC $60-272$ with various

4 OutD derivatives (indicated on top).

5 B. Co-purification assay on Glutathione Sepharose using the extracts shown in (A). Bound 6 proteins were eluted with Laemmli sample buffer. The proteins were separated by SDS-PAGE 7 and probed with Tetra-His antibodies and then with Ni-NTA conjugated with peroxidase. GST-OutC $\mathrm{C}_{60-272}$ is indicated by asterisk and OutD derivatives by triangles.

Fig. 5. Two distinct regions of OutD, OutD $\mathrm{D}_{28-112}$ and OutD $\mathrm{D}_{116-285}$, interact with the

\section{OutCsip peptide.}

A and B. Dissection of the OutCsip region interacting with $\operatorname{OutD}_{28-112}(\mathrm{~A})$ and $\operatorname{OutD}_{116-285}(\mathrm{~B})$. The GST-OutC derivatives (indicated at the top) were co-expressed with either OutD $116-285$ or proteins were eluted with Laemmli sample buffer, separated by SDS-PAGE and then stained with Coomassie G250 (middle panels) or probed with Tetra-His antibodies (lower panels).

C. Simultaneous binding of OutD $\mathrm{D}_{28-112}$ and $\mathrm{OutD}_{116-285}$ to $\mathrm{GST}-\mathrm{OutC}_{128-272}$. GST-OutC $128-272$ was co-expressed with either OutD $\mathrm{D}_{28-112}$ or OutD $\mathrm{D}_{116-285}$ or the three proteins were all expressed together and used in co-purification assays as above. GST-OutC derivatives are indicated by asterisks, Out $\mathrm{D}_{28-112}$ by open triangles and OutD $\mathrm{D}_{116-285}$ by filled triangles. Relative amounts of the proteins were estimated by densitometry and indicated as percentage of those with pairwise expressions (on bottom of the corresponding panels). The experiments were repeated three times and a representative assay is present. 
1 A. Stability of OutC mutant proteins (indicated on top) expressed in E. chrysanthemi A3618

$2 \Delta$ outC cells. Immunoblotting of whole cell extracts with OutC-antibodies. In the right panel,

3 three times more of material was loaded and the membrane was overexposed to detect low

4 abundance products. OutC, OutC $\mathrm{O}_{\Delta 163-169}$ and $\mathrm{OutC}_{\Delta 173-256}$ are indicated with a triangle. The

5 species of the molecular weight slightly larger than OutC is a cross-reaction product. Lower

6 molecular weight species in the OutC and $\mathrm{OutC}_{\Delta 163-169}$ lanes are degradation products.

7 B. Complementation ability of OutC mutant proteins. The supernatant (S) and cell extract (C)

8 of E. chrysanthemi A3618 $\triangle$ outC carrying a pTdB-oC plasmid with the corresponding mutant

9 outC (indicated on top) were analyzed by immunoblotting with PemA-antibodies.

11 Table 1. Strains used in this study

12

Strain

Genotype/phenotype

Reference

14

Escherichia coli

16

BL21(DE3)

$\mathrm{F}^{-}$dcm ompT hsdSB $\left(\mathrm{r}_{\mathrm{B}}{ }^{-}, \mathrm{m}_{\mathrm{B}}{ }^{-}\right)$gal lon $\lambda(\mathrm{DE} 3)$

Studier et al., 1990

17

BL21(DE3)C41

uncharacterized mutant of BL21(DE3)

Miroux \& Walker, 1996

$18 \quad$ NM522 supE thi-1 $\Delta($ lac-proAB $) \Delta($ mcrB-hsdSM $) 5$

Stratagene

Erwinia chrysanthemi 
A

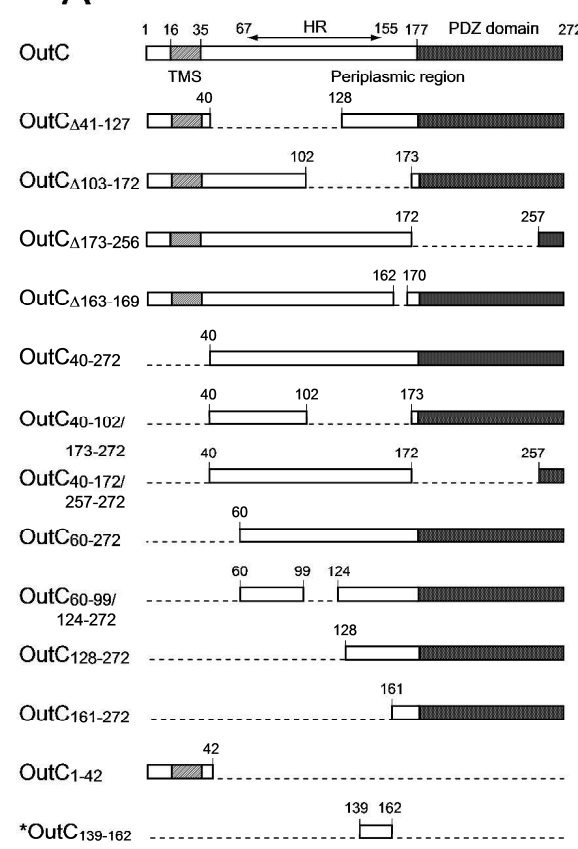

B

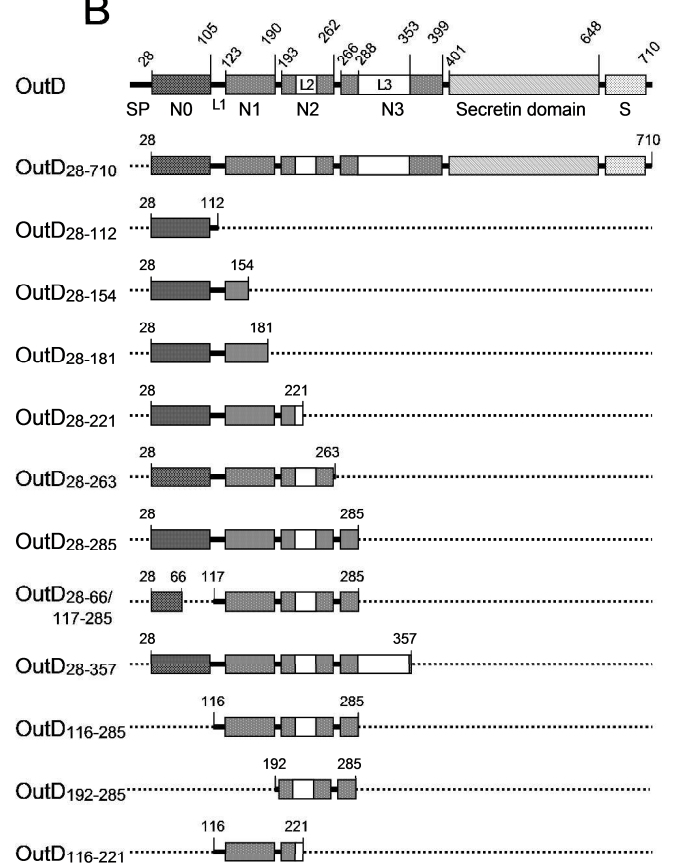




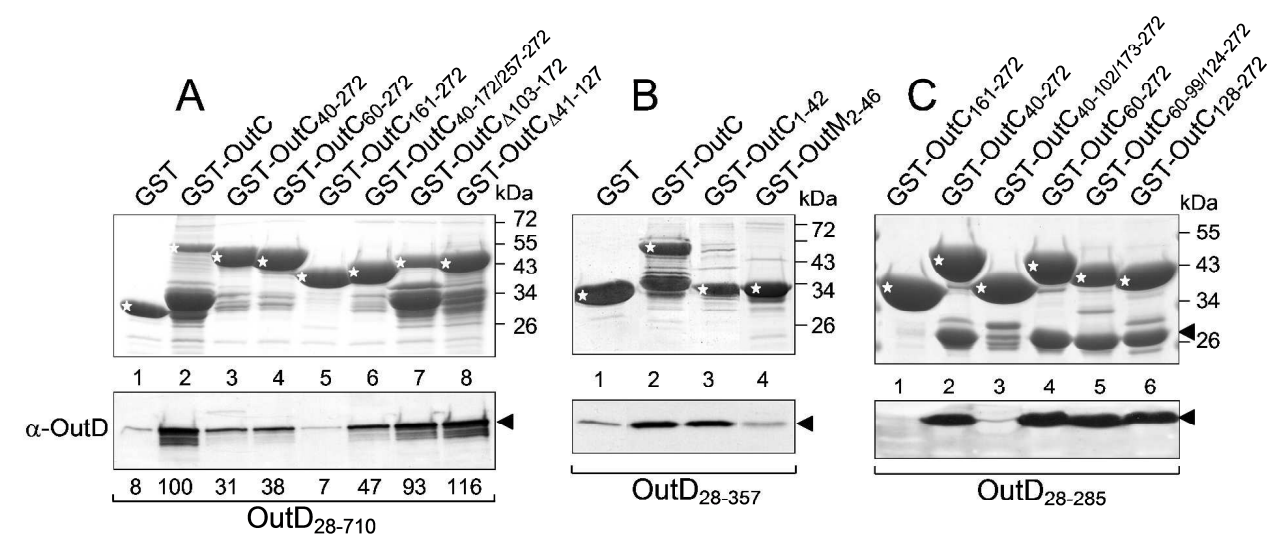

$1270 \times 952 \mathrm{~mm}(120 \times 120$ DPI $)$ 

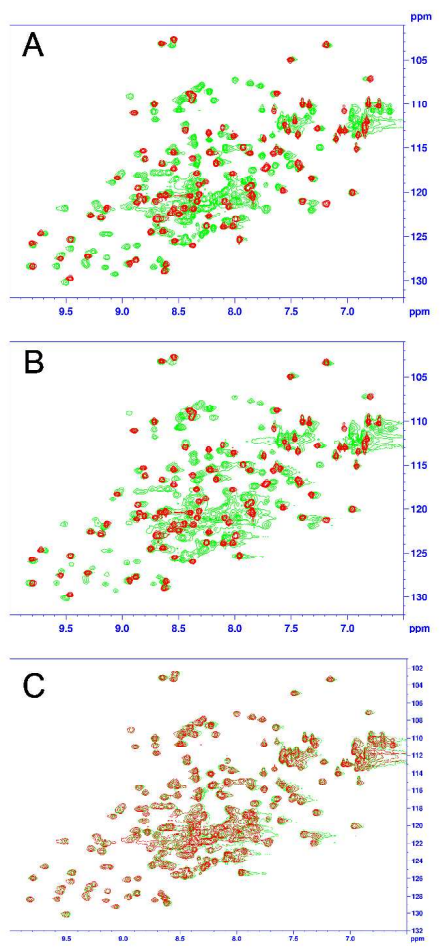

$1270 \times 952 \mathrm{~mm}(120 \times 120 \mathrm{DPI})$ 


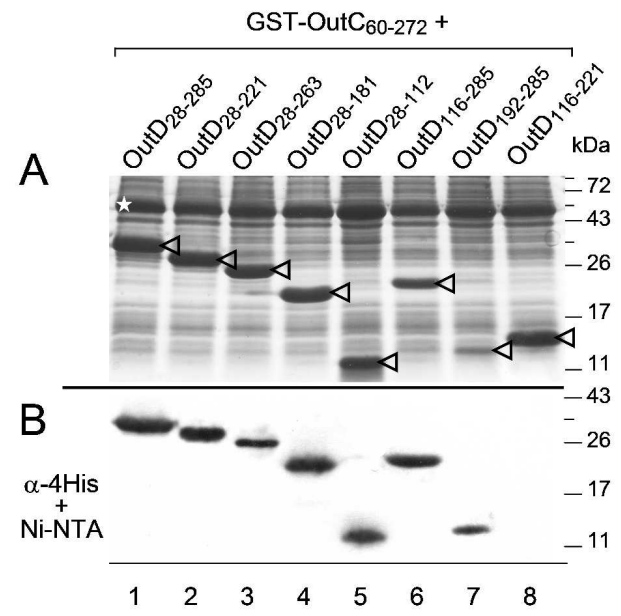

$1270 \times 952 \mathrm{~mm}(120 \times 120 \mathrm{DPI})$ 


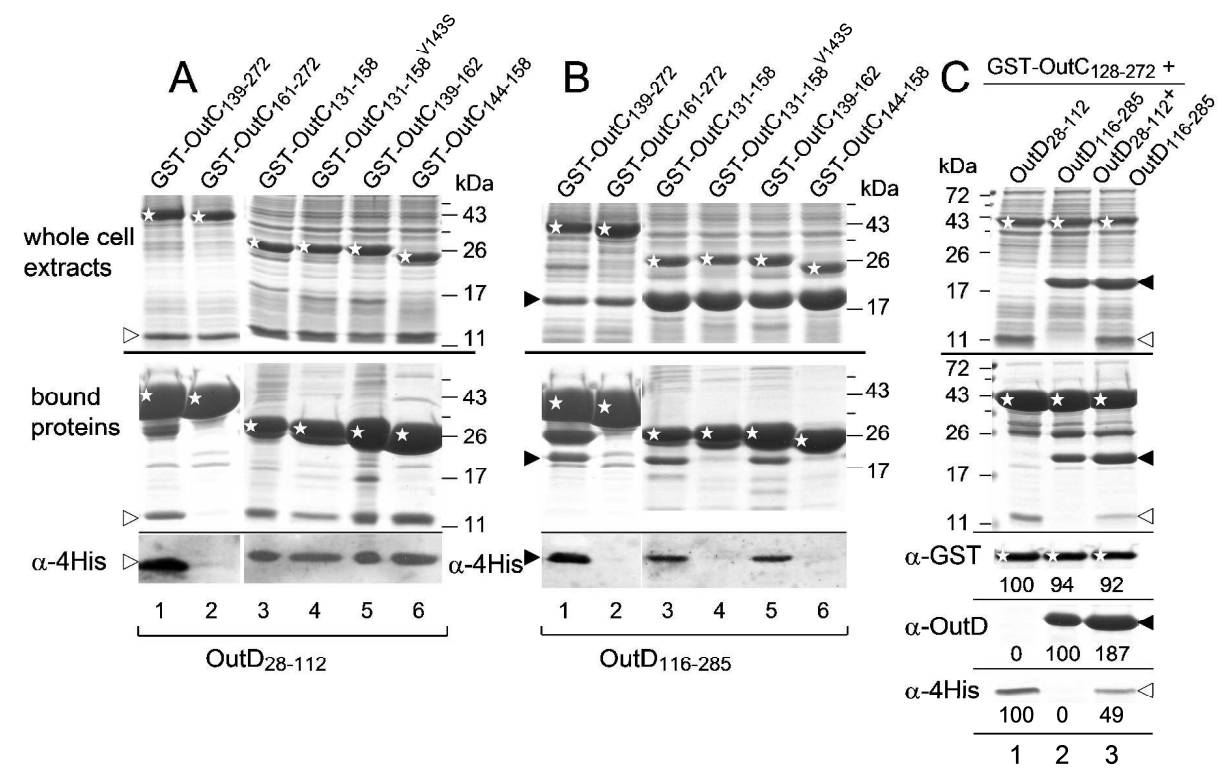

$1270 \times 952 \mathrm{~mm}(120 \times 120 \mathrm{DPI})$ 


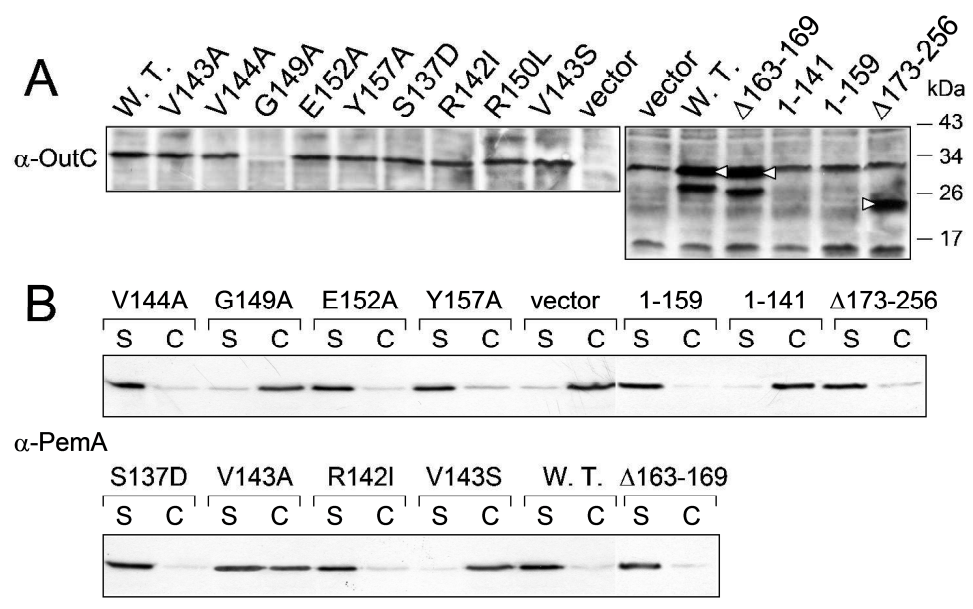

